\title{
A UNIFORM CONTRACTION PRINCIPLE FOR BOUNDED APOLLONIAN EMBEDDINGS
}

\author{
LOÏC DUBOIS AND HANS HENRIK RUGH
}

\begin{abstract}
AвStract. Let $\widehat{H}=H \cup\{\infty\}$ denote the standard one-point completion of a real Hilbert space $H$. Given any non-trivial proper subset $U \subset \widehat{H}$ one may define the so-called "Apollonian" metric $d_{U}$ on $U$. When $U \subset V \subset \widehat{H}$ are nested proper subsets we show that their associated Apollonian metrics satisfy the following uniform contraction principle: Let $\Delta=\operatorname{diam}_{V}(U) \in[0,+\infty]$ be the diameter of the smaller subsets with respect to the large. Then for every $x, y \in U$ we have

$$
d_{V}(x, y) \leq \tanh \frac{\Delta}{4} d_{U}(x, y)
$$

In dimension one, this contraction principle was established by Birkhoff [Bir57] for the Hilbert metric of finite segments on $\mathbb{R P}^{1}$. In dimension two it was shown by Dubois in [Dub09] for subsets of the Riemann sphere $\widehat{\mathbb{C}} \sim \widehat{\mathbb{R}^{2}}$. It is new in the generality stated here.
\end{abstract}

\section{INTRODUCTION AND RESUlts}

There are striking similarities between the projective group for the real or complex projective lines and the conformal group of the one-point completion of a real Hilbert space of dimension at least 3. In the first case, the group consists of Möbius maps of the form $z \mapsto \frac{a z+b}{c z+d}$ and in the second it is generated by linear isometries, homotheties and the inversion, corresponding to Möbius transformations supplemented with a complex conjugation. In both cases one needs at least 4 points to define a group invariant quantity, i.e. the cross-ratio. Fixing a subset $U$ whose complement contains at least 2 points, the logarithm of cross-ratios may then be used to construct a (semi-)metric on $U$. On the interval $I=(-1,1)$, there is a unique (up to a constant) distance invariant under Möbius transformations preserving $I$. This is precisely the restriction of the Poincaré metric $2|d z|\left(1-|z|^{2}\right)^{-1}$ on the unit disk in the complex plane. In the case of Hilbert spaces of higher dimensions one may derive the so-called 'Apollonian metric' (see below). This latter metric was first introduced for $\widehat{\mathbb{R}}^{n}=\mathbb{R}^{n} \cup\{\infty\}$ by Barbilian Bar34 and later rediscovered by Beardon [Bea98].

From a dynamical point of view, it is of interest to know how a subset $U$ metrically embed into a larger subset $V$ with respect to the associated metrics $d_{U}$

Received by the editors February 19, 2011.

2010 Mathematics Subject Classification. Primary 30F45, 53A30; Secondary 47H09, 30C35.

This research was partially funded by the European Research Council. 
and $d_{V}$ (see below for more precise statements). It is straight-forward from definitions that the injection $i:\left(U, d_{U}\right) \hookrightarrow\left(V, d_{V}\right)$ is non-expanding. More interesting, however, is that it verifies a general uniform contraction principle (UCP): If $\Delta=\operatorname{diam}_{V}(U)<+\infty$, i.e. the embedding of $U$ has bounded diameter in the larger domain $V$, then the injection is a strict contraction with a Lipschitz constant bounded by $\tanh \frac{\Delta}{4}$. This is the same formula which appears in Birkhoff's work on the Hilbert metric Bir57. We give below the (surprisingly simple) proof of the UCP for the general case and in section 2 some simple dynamical systems applications.

There is no particular reason for sticking to finite dimension, so in the following let $H$ be any real Hilbert space. We write $\langle\cdot, \cdot\rangle$ for the scalar product and $\|\cdot\|$ for the norm on $H$. Let $\widehat{H}=H \cup\{\infty\}$ be a one point completion of $H$ in which the open sets containing $\infty$ are of the form $\{\infty\} \cup F^{c}$ with $F$ a bounded closed set. With this convention $\widehat{H}$ is compact iff $H$ is finite dimensional. The space $(\widehat{H}, \widehat{d})$ is a complete metric space of diameter one with respect to the metric:

$$
\widehat{d}(x, y)=\frac{\|x-y\|}{\sqrt{1+\langle x, x\rangle} \sqrt{1+\langle y, y\rangle}}, \quad \widehat{d}(\infty, y)=\frac{1}{\sqrt{1+\langle y, y\rangle}} .
$$

Definition 1.1. Given four points $\left\{x_{1}, x_{2}, u_{1}, u_{2}\right\} \in \widehat{H}$ such that $\left\{x_{1}, x_{2}\right\}$ and $\left\{u_{1}, u_{2}\right\}$ are disjoint, we define their cross-ratio to be :

$$
\left[x_{1}, x_{2} ; u_{1}, u_{2}\right] \equiv \frac{\left\|x_{2}-u_{1}\right\|\left\|x_{1}-u_{2}\right\|}{\left\|x_{1}-u_{1}\right\|\left\|x_{2}-u_{2}\right\|} .
$$

Here, $\|\cdot\|$ denotes the Hilbert norm in $H$ and we adapt the usual conventions for dealing with the point at $\infty$. When $U \subset \widehat{H}$ is a proper subset (by proper we mean that $U$ and $U^{c}$ are both non-empty) one defines the Apollonian (semi-, pseudo-)distance between points $x_{1}, x_{2} \in U$ :

$$
d_{U}\left(x_{1}, x_{2}\right)=\sup _{u_{1}, u_{2} \in U^{c}} \log \left[x_{1}, x_{2} ; u_{1}, u_{2}\right] \in[0,+\infty] .
$$

We denote by $G M(\widehat{H})$ the general conformal group which acts continuously upon $(\widehat{H}, \widehat{d})$ and is generated by the set of isometries, homotheties (both fixing $\infty$ ) and the inversion (which exchanges the origin and $\infty$ ):

$$
I(x)=\frac{x}{\langle x, x\rangle}, \quad I(0)=\infty \quad \text { and } \quad I(\infty)=0
$$

When $\operatorname{dim} H \geq 3$, the Liouville theorem (see e.g. [Nev60]) shows that any conformal map is in $G M(\widehat{H})$. In dimensions 1 or 2, it is the Möbius group (supplemented with complex conjugation in the 2 dimensional case). That $d_{U}$ is $G M(\widehat{H})$ invariant is trivial for isometries and homotheties and in the case of inversions it follows easily from the formula $\|I(x)-I(y)\|=\frac{\|x-y\|}{\|x\|\|y\|}$ (with some care taken with respect to the point at infinity). From the cross-ratio identity $[x, z ; u, v]=[x, y ; u, v][y, z ; u, v]$ and taking sup in the right order, one also sees that $d_{U}$ verifies the triangular inequality. When $U^{c}$ has non-empty interior, $d_{U}$ is a genuine metric, but in the general case 
it need not distinguish points. We refer to e.g. Bea98, Chapter 3] and [Has04 for further details on the geometry of this metric. Our main result is the following:

Theorem 1.2 (Main Theorem). Let $U \subset V \subset \widehat{H}$ be non-empty proper subsets with $d_{U}$ and $d_{V}$ being the associated Apollonian metrics. Let $\Delta=\sup _{u_{1}, u_{2} \in U} d_{V}\left(u_{1}, u_{2}\right)$ be the diameter of the smaller subset within the larger. Then $\forall x_{1}, x_{2} \in U$ :

$$
d_{V}\left(x_{1}, x_{2}\right) \leq\left(\tanh \frac{\Delta}{4}\right) d_{U}\left(x_{1}, x_{2}\right) .
$$

If $\operatorname{diam}_{V}(U)<+\infty$, the embedding $i:\left(U, d_{V}\right) \hookrightarrow\left(U, d_{U}\right)$ is a uniform contraction.

Proof. We will base our proof upon Birkhoff's inequality Bir57 for cross-ratios on the projective real line. It is, in fact, a special case of our main theorem when $n=1$. We will use it in the following version: Let $K=\left(a_{1}, a_{2}\right)$ be a non-empty open sub-interval of $J=(0,+\infty)$. The Hilbert distance of $s_{1}, s_{2} \in K$ relative to $K$ and $J$ are given by:

$$
d_{K}\left(s_{1}, s_{2}\right)=\left|\log \left[s_{1}, s_{2} ; a_{1}, a_{2}\right]\right| \text { and } d_{J}\left(s_{1}, s_{2}\right)=\left|\log \frac{s_{2}}{s_{1}}\right| .
$$

The quantity $\Delta=\operatorname{diam}_{J}(K)=\log \frac{a_{2}}{a_{1}} \in(0,+\infty]$ measures the diameter of $K$ for the $J$-metric. Birkhoff [Bir57, p. 220] showed the fundamental inequality :

$$
d_{J}\left(s_{1}, s_{2}\right) \leq\left(\tanh \frac{\Delta}{4}\right) d_{K}\left(s_{1}, s_{2}\right), \quad \forall s_{1}, s_{2} \in K
$$

Proof of (1.6). It suffices to show this for $s_{1}$ and $s_{2}$ infinitesimally close. So we differentiate with respect to $s_{2}$ at $s_{2}=s_{1}=s \in\left(a_{1}, a_{2}\right)$ and search for the optimal value of $\theta>0$ so that for every $a_{1}<s<a_{2}: \frac{1}{s} \leq \theta \frac{a_{1}-a_{2}}{\left(s-a_{1}\right)\left(a_{2}-s\right)}$, or equivalently

$$
\theta \geq \inf _{a_{1}<s<a_{2}} \frac{\left(s-a_{1}\right)\left(a_{2}-s\right)}{s\left(a_{2}-a_{1}\right)} .
$$

The minimum value at $s=\sqrt{a_{1} a_{2}}$ is $\theta_{\min }=\frac{\sqrt{a_{2}}-\sqrt{a_{1}}}{\sqrt{a_{2}}+\sqrt{a_{1}}}=\tanh \frac{\log \left(a_{2} / a_{1}\right)}{4}$ which is therefore the desired contraction constant.

Now, returning to the general case, let $x_{1}, x_{2} \in U$ be distinct points. We have $d_{V}\left(x_{1}, x_{2}\right) \leq d_{U}\left(x_{1}, x_{2}\right)$ since the sup in the latter case is over a larger set. We may assume that $\Delta=\operatorname{diam}_{V}(U)<+\infty$ and $0<d_{V}\left(x_{1}, x_{2}\right) \leq d_{U}\left(x_{1}, x_{2}\right)<+\infty$ (or else the statement is trivial). Let $\epsilon>0$ and pick $v_{1}, v_{2} \in V^{c}$ so that

$$
d_{V}\left(x_{1}, x_{2}\right) \leq(1+\epsilon) \log \left[x_{1}, x_{2} ; v_{1}, v_{2}\right] .
$$

To simplify calculations, we choose a transformation in $G M(\widehat{H})$ which maps $v_{1}$ to zero and $v_{2}$ to infinity. We recall that this preserves cross-ratios. By a slight abuse of notation we still write $x_{1}, x_{2}$ for the images in $\widehat{H}$ of the corresponding points. We have then $0<d_{V}\left(x_{1}, x_{2}\right) \leq(1+\epsilon) \log \frac{\left\|x_{2}\right\|}{\left\|x_{1}\right\|}$ so, in particular, $\left\|x_{1}\right\|<\left\|x_{2}\right\|$. When 


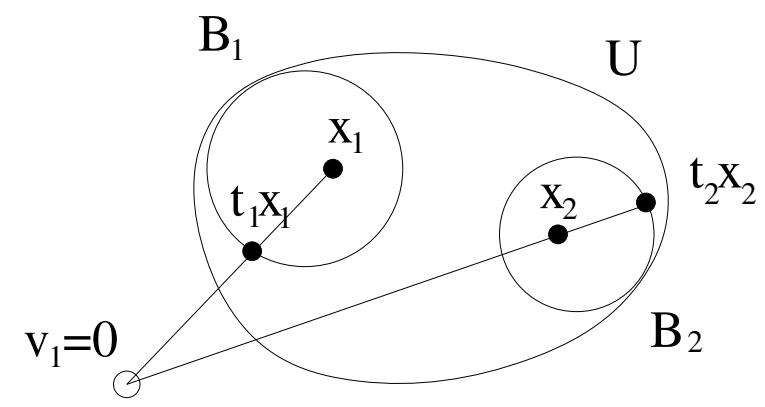

FiguRE 1. Construction of cross-ratios. $v_{1}=0$ and $v_{2}=\infty$.

$u_{1}, u_{2} \in U$ we have, in these new coordinates,

$$
\left|\log \frac{\left\|u_{2}\right\|}{\left\|u_{1}\right\|}\right|=\left|\log \left[u_{1}, u_{2}, 0, \infty\right]\right| \leq d_{V}\left(u_{1}, u_{2}\right) \leq \Delta<+\infty .
$$

In other words, $U$ is bounded away from the origin and infinity.

Consider now the formula for the distance of $x_{1}, x_{2}$ relative to $U$. It splits into a sum of two supremums (this splitting is one of the deeper reasons why the Apollonian metric is easy to handle):

$$
d_{U}\left(x_{1}, x_{2}\right)=\sup _{u_{1} \in U^{c}} \log \frac{\left\|x_{2}-u_{1}\right\|}{\left\|x_{1}-u_{1}\right\|}+\sup _{u_{2} \in U^{c}} \log \frac{\left\|x_{1}-u_{2}\right\|}{\left\|x_{2}-u_{2}\right\|} .
$$

The suprema of these two terms are denoted $\alpha_{1}$ and $\alpha_{2}$. They are both finite. We define the Apollonian ball

$$
B_{1}=B_{\alpha_{1}}\left(x_{1}, x_{2}\right)=\left\{u \in \widehat{H}: \frac{\left\|x_{1}-u\right\|}{\left\|x_{2}-u\right\|}<\alpha_{1}\right\} \subset U
$$

and similarly for the ball $B_{2}=B_{\alpha_{2}}\left(x_{2}, x_{1}\right) \subset U$ (see Figure 1).

A priori $B_{1}$ is a generalized open ball containing $x_{1}$ but as $U$ is bounded, $B_{1}$ must be an open ball in the usual bounded sense (and $\alpha_{1}$ must be greater than one). Let $t_{1} x_{1}$ (with $0<t_{1}<1$ ) be the intersection between the segment $\left\{t x_{1}: 0 \leq t \leq 1\right\}$ and the sphere $\partial B_{u_{1}}\left(x_{1}, x_{2}\right)$. Similarly, let $t_{2} x_{2}$ (with $1<t_{2}<+\infty$ ) be the intersection between the segment $\left\{t \quad x_{2}: 1 \leq t \leq+\infty\right\}$ and $\partial B_{u_{2}}\left(x_{2}, x_{1}\right)$ (see Figure (1). Then $t_{1} x_{1}, t_{2} x_{2} \in \mathrm{Cl} U$ and $\left\|t_{1} x_{1}\right\|<\left\|x_{1}\right\|<\left\|x_{2}\right\|<\left\|t_{2} x_{2}\right\|$. From the way we defined $t_{1}$ and $t_{2}$ we have the following lower bound

$$
\begin{aligned}
d_{U}\left(x_{1}, x_{2}\right)=\alpha_{1}+\alpha_{2} & =\log \frac{\left\|x_{2}-t_{1} x_{1}\right\|}{\left\|x_{1}-t_{1} x_{1}\right\|} \times \frac{\left\|x_{1}-t_{2} x_{2}\right\|}{\left\|x_{2}-t_{2} x_{2}\right\|} \\
& =\log \frac{\left\|x_{2}-t_{1} x_{1}\right\|}{\left\|x_{1}\right\|-\left\|t_{1} x_{1}\right\|} \times \frac{\left\|x_{1}-t_{2} x_{2}\right\|}{\left\|t_{2} x_{2}\right\|-\left\|x_{2}\right\|} \\
& \geq \log \frac{\left\|x_{2}\right\|-\left\|t_{1} x_{1}\right\|}{\left\|x_{1}\right\|-\left\|t_{1} x_{1}\right\|} \times \frac{\left\|t_{2} x_{2}\right\|-\left\|x_{1}\right\|}{\left\|t_{2} x_{2}\right\|-\left\|x_{2}\right\|} .
\end{aligned}
$$

The last expression is the cross-ratio of the four (ordered) points on the positive real line $0<\left\|t_{1} x_{1}\right\|<\left\|x_{1}\right\|<\left\|x_{2}\right\|<\left\|t_{2} x_{2}\right\|<+\infty$. Let us write $J=(0, \infty)$, $K=\left(\left\|t_{1} x_{1}\right\|,\left\|t_{2} x_{2}\right\|\right)$ and $s_{1}=\left\|x_{1}\right\|, s_{2}=\left\|x_{2}\right\|$. By our construction

$$
\operatorname{diam}_{J}(K)=\log \frac{\left\|t_{2} x_{2}\right\|}{\left\|t_{1} x_{1}\right\|} \leq d_{V}\left(t_{1} x_{1}, t_{2} x_{2}\right) \leq \operatorname{diam}_{V}(U)=\Delta
$$


where we used that $t_{1} x_{1}, t_{2} x_{2} \in \mathrm{Cl} U$ and that $v_{1}=0, v_{2}=\infty \in V$. Also, $d_{K}\left(\left\|x_{1}\right\|,\left\|x_{2}\right\|\right) \leq d_{U}\left(x_{1}, x_{2}\right)$ by the above bound (1.8). So using Birkhoff's inequality (1.6) we get

$$
\frac{d_{V}\left(x_{1}, x_{2}\right)}{1+\epsilon} \leq d_{J}\left(s_{1}, s_{2}\right) \leq\left(\tanh \frac{\operatorname{diam}_{J}(K)}{4}\right) d_{K}\left(s_{1}, s_{2}\right) \leq\left(\tanh \frac{\Delta}{4}\right) d_{U}\left(x_{1}, x_{2}\right),
$$

and since $\epsilon>0$ was arbitrary we see that $d_{V}\left(x_{1}, x_{2}\right) \leq\left(\tanh \frac{\Delta}{4}\right) d_{U}\left(x_{1}, x_{2}\right)$, which is what we aimed to show.

\section{Some APPlications}

In the one dimensional case, the result of Birkhoff [Bir57] has a vast variety of applications related to the Perron-Frobenius type of results and the presence of spectral gaps of real operators contracting a real convex cone; see e.g. [Bal00. In the case of complex operators similar spectral gap results were obtained first in Rug10 and then simplified in Dub09 using a complex Hilbert metric and the 2-dimensional version of the UCP for the Apollonian metric. We discuss in the following some possible applications in the case of arbitrary dimension.

Corollary 2.1. Let $U \subset V$ and $\Delta$ be as in the main theorem and write $\Gamma(V, U)=$ $\{\gamma \in G M(\widehat{H}): \gamma(V) \subset U\}$ for the elements of the conformal group that map $V$ into $U$. Then for every $\gamma \in \Gamma(V, U)$ we have $\gamma^{-1} \in \Gamma\left(U^{c}, V^{c}\right)$ and the mappings $\gamma:\left(V, d_{V}\right) \rightarrow\left(V, d_{V}\right)$ and $\gamma^{-1}:\left(U^{c}, d_{U^{c}}\right) \rightarrow\left(U^{c}, d_{U^{c}}\right)$ are $\left(\tanh \frac{\Delta}{4}\right)$-Lipschitz.

Proof. $\gamma \in \Gamma(U, V)$ preserves cross-ratios, and $\gamma(V) \subset U$ so writing $\theta=\tanh \Delta / 4$ we have for $v_{1}, v_{2} \in V$ :

$$
d_{V}\left(\gamma\left(v_{1}\right), \gamma\left(v_{2}\right)\right) \leq \theta d_{U}\left(\gamma\left(v_{1}\right), \gamma\left(v_{2}\right)\right) \leq \theta d_{\gamma(V)}\left(\gamma\left(v_{1}\right), \gamma\left(v_{2}\right)\right) \leq \theta d_{V}\left(v_{1}, v_{2}\right) .
$$

The inverse map is bijective so it maps $U^{c}$ into $V^{c}$. We have the same bound for its contraction rate since

$$
\operatorname{diam}_{V}(U)=\operatorname{diam}_{U^{c}}\left(V^{c}\right)=\sup _{v_{1}, v_{2} \in V} \sup _{u_{1}, u_{2} \in U^{c}} \log \left[u_{1}, u_{2} ; v_{1}, v_{2}\right] .
$$

Corollary 2.2. In finite dimension when $\mathrm{Cl} U \subset \operatorname{Int} V$ for the topology of $(\widehat{H}, \widehat{d})$, compactness shows that $\operatorname{diam}_{V}(U)<+\infty$ so the embedding $\left(U, d_{U}\right) \hookrightarrow\left(V, d_{V}\right)$ is a strict Lipschitz contraction.

Lemma 2.3. Suppose that $U \subset B\left(x_{0}, R\right), R<\infty$. Then

$$
\left\|u_{1}-u_{2}\right\| \leq \frac{R}{2} d_{U}\left(u_{1}, u_{2}\right), \quad \forall u_{1}, u_{2} \in U .
$$

Suppose that $U \subset V$ and that $r=\operatorname{dist}\left(U, V^{c}\right)=\sup _{u \in U, w \in V^{c}}\|u-w\|>0$. Then

$$
d_{V}\left(u_{1}, u_{2}\right) \leq \frac{2}{r}\left\|u_{1}-u_{2}\right\|, \quad \forall u_{1}, u_{2} \in U .
$$

Proof. When $x \in B\left(x_{0}, R\right)$ and $h$ is small we get from a straight-forward calculation:

$$
d_{B}(x, x+h)=\frac{2 R}{R^{2}-\left\|x-x_{0}\right\|^{2}}\|h\|+o(h) .
$$

Thus, $d s=\frac{2 R}{R^{2}-\left\|x-x_{0}\right\|^{2}}\|d x\| \geq \frac{2}{R}\|d x\|$ and $\left\|v_{1}-v_{2}\right\| \leq d_{B}\left(v_{1}, v_{2}\right) \leq d_{V}\left(v_{1}, v_{2}\right)$ (since $V \subset B$ ). 
If $B\left(u_{1}, r\right), B\left(u_{2}, r\right) \subset V$, then we get for $w \in V^{c}: \frac{\left\|u_{2}-w\right\|}{\left\|u_{1}-w\right\|} \leq 1+\frac{\left\|u_{2}-u_{1}\right\|}{r}$ and $d_{V}\left(u_{1}, u_{2}\right) \leq 2 \log \left(1+\frac{\left\|u_{2}-u_{1}\right\|}{r}\right) \leq \frac{2}{r}\left\|u_{2}-u_{1}\right\|$.

Theorem 2.4. Let $U \subset V$ be non-empty proper subsets of $(\widehat{H}, \widehat{d})$ such that $\mathrm{Cl} V \neq$ $\widehat{H}$ and $\Delta=\operatorname{diam}_{V}(U)<+\infty$. Let $\gamma_{1}, \ldots, \gamma_{k} \in \Gamma(V, U)$ and write

$$
\Lambda \equiv \Lambda\left(\gamma_{1}, \ldots, \gamma_{k}\right)=\bigcap_{n \geq 1} \mathrm{Cl} \bigcup_{1 \leq i_{1}, \ldots, i_{n} \leq k} \gamma_{i_{1}} \circ \cdots \circ \gamma_{i_{n}}(V)
$$

for the associated limit set. Then $\Lambda$ is compact and has Hausdorff and Box dimensions not greater than $-\log k / \log \tanh \frac{\Delta}{4}$.

Proof. Pick $q \in \widehat{H} \backslash \mathrm{Cl} V$ and map $q$ to infinity by an inversion in $q$. In the new coordinates, $V$ is bounded, so by the previous lemma, Hilbert distances are bounded by Apollonian distances. At level $n \geq 1$ each set in the finite union has diameter not greater than $r=\Delta\left(\tanh \frac{\Delta}{4}\right)^{n-1}$ which becomes arbitrarily small as $n \rightarrow \infty$. There are $N_{r}=k^{n}$ elements in the union. As $\Lambda$ is closed and has finite covers of arbitrarily small diameters it is compact and we have the bound

$$
\operatorname{dim}_{H}(\Lambda) \leq \limsup _{n} \frac{\log N_{r}}{\log 1 / r}=\frac{\log k}{\log \tanh \frac{\Delta}{4}} .
$$

When the images $\mathrm{Cl}\left(\gamma_{i}(V)\right), 1 \leq i \leq k$ are pairwise disjoint, the Hausdorff dimension may also be obtained from a Bowen-like formula as in Rug08 or [MU98]. We omit the details. Note that we do not assume here that $H$ is finite dimensional.

Remark 2.5. In finite dimension $d \geq 2$ the Apollonian metric for an open ball $V=B(0, R)$ is the same as the hyperbolic metric for the ball, i.e. $d s=\frac{2 r\|d x\|}{r^{2}-\|x\|^{2}}$. In this case it is well known that if $\gamma$ maps $V$ inside $V$ and $\gamma(V)$ has bounded diameter, then $\gamma$ is a uniform contraction.

Other metrics may be constructed from the Apollonian metric (cf. Has04]). Let $v \in H^{*},\|h\| \leq\|v\| / 4$ and write $x=\frac{\langle h, v\rangle}{\langle v, v\rangle} \in[-1 / 4,1 / 4]$ and $\|h\|^{2}=\|v\|^{2}\left(x^{2}+y^{2}\right)$. Calculus shows that $\left|\frac{1}{2} \log \left((1+x)^{2}+y^{2}\right)-x\right| \leq x^{2}+y^{2}$ (when $\left.x \geq-1 / 4\right)$. Therefore,

$$
\left|\log \frac{\|v+h\|}{\|v\|}-\langle I(v), h\rangle\right|=\left|\log \frac{\|v+h\|}{\|v\|}-\frac{\langle v, h\rangle}{\langle v, v\rangle}\right| \leq \frac{\langle h, h\rangle}{\langle v, v\rangle} .
$$

Assume in the following that $U$ is open. Let $x \in U$ and set $r=\inf _{u \in U^{c}} d(x, u)>0$. When $u_{1}, u_{2} \in U^{c}$ and $\|h\| \leq r / 4$ we get:

$$
\left|\log \left[x, x+h ; u_{1}, u_{2}\right]-\left\langle I\left(x-u_{1}\right)-I\left(x-u_{2}\right), h\right\rangle\right| \leq 2\|h\|^{2} / r^{2} .
$$


It follows that the following limit exists and define a Finsler (pseudo-)norm on the tangent space of $U$ :

$$
p_{U, x}(h) \equiv \lim _{t \rightarrow 0} \frac{1}{t} d_{U}(x+t h, x)=\sup _{u_{1}, u_{2} \in U^{c}}\left|\left\langle I\left(x-u_{1}\right)-I\left(x-u_{2}\right), h\right\rangle\right| .
$$

It is only a pseudo-norm when $U^{c}$ is contained in a generalized ball, since in that case $p_{U, x}$ may vanish in some directions. If $\gamma:[0,1] \rightarrow U$ is a continuous path, then we may define its (pseudo-)length to be

$$
\ell(\gamma) \equiv \limsup _{\delta \rightarrow 0} \sum_{k=0}^{n} d_{U}\left(\gamma\left(t_{k+1}\right), \gamma\left(t_{k}\right)\right),
$$

where $0=t_{0}<t_{1}<\cdots<t_{n}=1$ and $t_{k+1}-t_{k}<\delta$. Then

$$
d_{U}^{\operatorname{in}}(x, y)=\inf \{\ell(\gamma): \gamma \in C([0,1], U), \gamma(0)=x, \gamma(1)=y\}
$$

defines a (pseudo-)metric which in [Has04] was coined the Apollonian inner metric. When $\gamma$ is piecewise $C^{1}$, we have $\ell(\gamma)=\int_{0}^{1} p_{U, x}(\dot{\gamma}(t)) d t$. Another possiblity is to maximize (2.3) over directions. This leads to a conformal Riemannian metric $d s=g_{U}(x)\|d x\|$ with

$$
g_{U}(x)=\sup _{\|h\|=1} p_{U, x}(h)=\sup _{u_{1}, u_{2} \in U^{c}} \frac{\left\|u_{1}-u_{2}\right\|}{\left\|x-u_{1}\right\|\left\|x-u_{2}\right\|} .
$$

An advantage of this metric is perhaps that it distinguishes points when $U^{c}$ contains at least two points. It is easy to see that $g_{U}(x)$ is continuous (as we assumed $U$ to be open). We write $d_{U}^{\mathrm{Rie}}(x, y)$ for the Riemannian distance of $x$ and $y$ with respect to this metric.

Corollary 2.6. Let $U \subset V \subset \widehat{H}$ (with $\mathrm{Cl} V \neq \widehat{H}$ ) be non-empty proper subsets and let $\Delta=\sup _{u_{1}, u_{2} \in U} d_{V}\left(u_{1}, u_{2}\right)$ be the diameter of the smaller subset within the larger with respect to the Apollonian metric. Then for every $x, y \in U$ :

$$
\begin{gathered}
p_{V, x}(h) \leq\left(\tanh \frac{\Delta}{4}\right) p_{U, x}(h), \quad h \in E, \\
d_{V}^{\mathrm{in}}(x, y) \leq\left(\tanh \frac{\Delta}{4}\right) d_{U}^{\mathrm{in}}(x, y), \\
d_{V}^{\mathrm{Rie}}(x, y) \leq\left(\tanh \frac{\Delta}{4}\right) d_{U}^{\mathrm{Rie}}(x, y) .
\end{gathered}
$$

Proof. For $x, x+t h \in U$ we have by the Main Theorem

$$
\frac{1}{t} d_{V}(x, x+t h) \leq \tanh \frac{\Delta}{4} \frac{1}{t} d_{U}(x, x+t h) .
$$

The first inequality follows. The second follows by taking limits in the right order. For the Riemmannian metric, one has

$$
g_{V}(x) \leq \sup _{\|h\|=1} p_{U, x}(h) \leq \sup _{\|h\|=1}\left(\tanh \frac{\Delta}{4}\right) p_{U, x}(h)=\left(\tanh \frac{\Delta}{4}\right) g_{U}(x)
$$

which yields the last inequality. 


\section{REFERENCES}

[Bal00] V. Baladi, Positive transfer operators and decay of correlations, World Sci Publ. (2000). MR.1793194 (2001k:37035)

[Bar34] D. Barbilian, Einordnung von Lobatchewsky's Maßbestimmung in gewisse allgemeine Metric der Jordanschen Bereiche, Casopsis Mathematiky a Fysiky, 64, 182-183 (1934-35).

[Bea95] A.F. Beardon, The Geometry of Discrete Groups, 2nd edn., Springer (1995). MR $1393195(97 \mathrm{~d}: 22011)$

[Bea98] A.F. Beardon, The Apollonian metric of a domain in $\mathbb{R}^{n}$, in "Quasiconformal Mappings and Analysis", Springer, New York, 91-108 (1998). MR1488447(99k:30075)

[Bir57] G. Birkhoff, Extensions of Jentzsch's theorem, Trans. Amer. Math. Soc., 85, 219-227 (1957). MR0087058 (19:296a)

[Cay1859] A. Cayley, A sixth memoirs upon quantics, Phil. Trans. Royal Soc. of London 149, 61-90, Collected Math Papers, vol. 2 (1859).

[Dub09] L. Dubois, Projective metrics and contraction principles for complex cones, J. London Math. Soc. 79, 719-737 (2009). MR2506695 (2010g:47160)

[Has04] P.A. Hästö, The Apollonian Inner Metric, Comm. An. and Geom., 12, no. 4, 927-947 (2004). MR2104081 (2006e:30050)

[Hil1895] D. Hilbert, Uber die gerade Linie als kürzeste Verbindung zweier Punkte. (Aus einem an Herrn F. Klein gerichten Briefe), Math. Ann., 46, 91-96 (1895).

[MU98] Mauldin, R.D., Urbanski, M., Dimension and measures for a curvilinear Sierpinski gasket or Apollonian packings, Advances in Mathematics, 136, 26-38 (1998). MR:1623671 (99h:28015)

[Nev60] R. Nevanlinna, Analytic functions, Princeton Mathematical Series, Number 24, Princeton, 1960.

[Rug08] H.H. Rugh, On the dimensions of conformal repellers. Randomness and parameter dependency, Ann. Math., 168, no. 3, 695-748 (2008). MR2456882(2010b:37131)

[Rug10] H. H. Rugh, Cones and gauges in complex spaces: Spectral gaps and complex PerronFrobenius theory, Ann. Math., 171, no. 3, 1702-1752 (2010). MR2680397

Department of Mathematics and Statistics, University of Helsinki, Finland Fi-00014

E-mail address: loic.dubois@helsinki.fi

University of Cergy-Pontoise, CNRS UMR 8088, France.

E-mail address: hhrugh@math.u-cergy.fr 